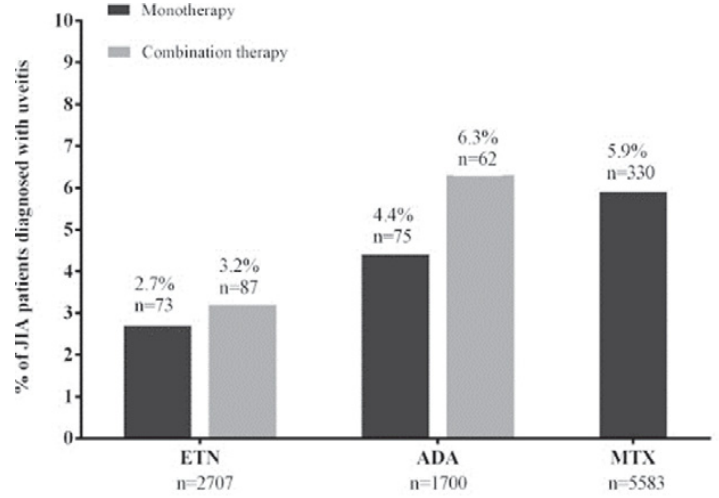

Note: Combination refers to ETN + MTX or ADA + MTX

Abbreviations: JIA, juvenile idiopathic arthritis; ETN, etanercept; ADA, adalimumab; MTX methotrexate

Figure 1. To show the patients with JIA receiving a monotherapy (with ETN, ADA or MTX) or a combination therapy and the number of uveitis diagnoses.

DOI: 10.1136/annrheumdis-2017-eular.1857

\section{THU0520 PELVIC ULTRASOUND IN THE ASSESSMENT OF SEXUAL MATURITY IN GIRLS WITH JUVENILE IDIOPATHIC ARTHRITIS}

L.M. Gonçalves ${ }^{1}$, S.H. Machado ${ }^{2}$, R.M. Xavier ${ }^{3}$, P.J.C. Maróstica ${ }^{4}$, C. Kopacek ${ }^{5}$, I.R.S. Lucena $6 .{ }^{1}$ Student, PUCRS; ${ }^{2}$ Pediatrics rheumatology, HCPA; ${ }^{3}$ Rheumatology service: ${ }^{4}$ Pediatrics service, Hcpa/UFRGS;

${ }^{5}$ Endocrinology service, HCSA; ${ }^{6}$ Radiology service, HCPA, Porto Alegre, Brazil

Background: Delayed puberty is common in children with chronic illnesses such as Juvenile Idiopathic Arthritis (JIA),especially in cases with an earlier age of onset.lts etiology is multifactorial and includes low weight,complications arising from the disease itself and the adverse effects of treatment.

Objectives: To compare uterine and ovarian size as well as artery pulsatility between girls with juvenile idiopathic arthritis (JIA) and healthy participants using abdominal pelvic ultrasound,and identify these findings and pubertal staging,sex hormones and disease characteristics in girls with JIA.

Methods: This study involved 44 girls with JIA and 59 healthy controls aged between six and 18 incomplete years. Pelvic ultrasound was used to determine uterine volume and length,the corpus/cervix ratio,ovarian volume and length,and the pulsatility index $(\mathrm{PI})$ of uterine arteries. Hormone levels were also measured in girls with JIA.

Results: All US parameters were associated with Tanner stages in the control group $(p<0.001)$.Uterine and ovarian measures were smaller in girls with JIA

Table 1. Comparison of abdominal pelvic US findings between control participants and patients with JIA

\begin{tabular}{lccc}
\hline Variables $^{*}$ & Patients $(\mathrm{n}=? 44)$ & Control participants $(\mathrm{n}=? 59)$ & $\mathrm{p}$ \\
\hline Uterine volume $\left(\mathrm{cm}^{3}\right)$ & $10.5(2.4-40)$ & $36.6(8.8-50.9)$ & 0.009 \\
Uterine length $(\mathrm{cm})$ & $4.92 \pm 1.83$ & $5.91 \pm 1.80$ & 0.008 \\
Corpus to cervix ratio $(\mathrm{cm})$ & $1.18 \pm 0.25$ & $1.36 \pm 0.27$ & 0.001 \\
Endometrium $(\mathrm{mm})$ & $1.4(0-5.2)$ & $5.4(1.4-7.2)$ & 0.016 \\
Left Ovarian Length $(\mathrm{cm})$ & $2.60 \pm 0.69$ & $3.04 \pm 0.84$ & 0.006 \\
Left Ovarian Volume $\left(\mathrm{cm}^{3}\right)$ & $3.3(1.5-5.2)$ & $5.0(3.0-7.1)$ & 0.004 \\
Right Ovarian Length $\left(\mathrm{cm}^{3}\right)$ & $2.79 \pm 0.71$ & $3.17 \pm 0.75$ & 0.013 \\
Right Ovarian Volume $\left(\mathrm{cm}^{3}\right)$ & $2.9(1.5-6.3)$ & $5.9(3.0-9.1)$ & 0.006 \\
Mean uterine PI & $4.6(2.7-6.5)$ & $2.5(1.9-5.1)$ & 0.025 \\
\hline
\end{tabular}

$\mathrm{PI}$, pulsatility index. ${ }^{*}$ Described as median (25th-75th percentile) or mean \pm standard deviation.

Figure 1 - Comparison of uterine volume between groups across different age ranges

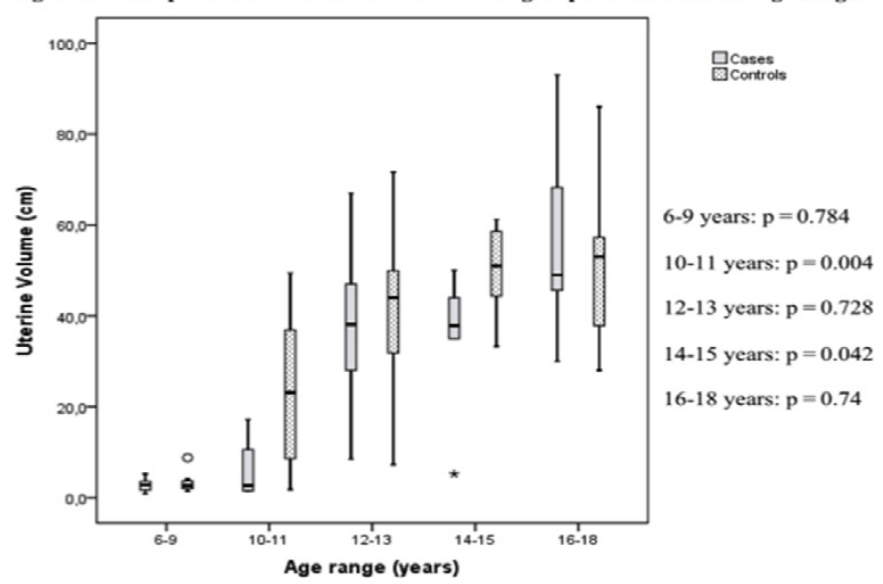

than in the control group.The mean PI of uterine arteries was higher in girls with JIA.Comparisons by age group revealed that uterine volumes were smaller in girls with JIA aged between 10 and 11 years $(p=0.004)$ and 14 to 15 years $(p=0.042)$, and the corpus/cervix ratio was smaller in girls aged 10 to 11 years $(p=0.007)$.US measures were not associated with disease factors in the JIA group.LH and estradiol levels were found to be positively associated with ovarian and uterine size $(p<0.001)$, but negatively correlated with the mean PI of uterine arteries $(p<0.01)$.

Conclusions: Pelvic US is a sensitive method for the assessment of sexual maturation in girls, and can identify developmental delays in girls with JIA which may not be detected by Tanner staging.

References:

[1] Pozo J,Argente J.Delayed puberty in chronic illness.Best Pract Res Clin Endocrinol Metab 2002;16:73-90.

[2] Holm K,Laursen EM,Brocks V,Muller J.Pubertal maturation of internal genitalia:authors' reply.Ultrasound Obstet Gynecol 1995;6:452-3.

Disclosure of Interest: None declared

DOI: 10.1136/annrheumdis-2017-eular.4389

\section{THU0521 ENTHESITIS-RELATED ARTHRITIS: NON-PERIPHERAL PATTERN IS ASSOCIATED WITH TH17 CELLS}

M. Katsicas, C. Carrara, A. Bernasconi, J. Rossi, R. Russo. Immunology \& Rheumatology, Hospital de Pediatría Garrahan, Buenos Aires, Argentina

Background: Enthesitis-related arthritis (ERA) is a category of juvenile idiopathic arthritis. Different proinflammatory cytokines linked to the Th1 and Th17 T- cell subsets have been implicated in its pathogenesis. Limited data are currently available about the relationship between disease activity and the clinical pattern and the percentage of Th1 and Th17 T- cell subsets.

Objectives: To analyze Th1 and Th17 cell subsets in patients with ERA and to compare with age-matched healthy controls. To assess the association between disease activity and disease clinical pattern with Th1and Th17 cells subsets

Methods: Patients with ERA (according to ILAR criteria) were included in a cross sectional study. Disease activity measures were collected in random visits: active joint count $(A J)$, pain score $(0-10)$, presence of active enthesitis (AE), sacroiliac pain (SIP), lumbar pain (LP),lumbar limitation (LL) by Schöber's test, wellbeing according to the patient using a visual analogue scale (VASp, 0-10), disease activity according to the physician (VASphy, 0-10), JADAS-10, JSpADA, and ESR/CRP were evaluated. Patients were classified based on the disease pattern (peripheral and non-peripheral) depending on the presence or absence of $\mathrm{AJ}$ and/or AE. Functional capacity was also assessed by $\mathrm{CHAQ}$. Presence of radiologic sacroiliitis (MRI/X-rays) and treatment with TNF inhibitors (TNFi) were recorded. Th-17 and Th-1 cells were quantified by flow cytometry in PBMCs stimulated with PMA/IO. Age-matched healthy children without disease or medication were recruited as normal control. Comparison between groups (Mann-Whitney Utest) and correlation tests as appropiate

Results: Twenty-nine patients ( $90 \%$ M) fulfilled inclusion criteria. HLA-B27 was positive in $13(45 \%)$. Median age at observation was 12 years and median disease duration was 2.1 years. Activity and functional measures were (medians): AJ 1, pain 0.25, VASp 0.5, VASphy 1, JADAS-10 7, JSpADA 1.75, ESR 15 $\mathrm{mm} / \mathrm{h}$, and $\mathrm{CHAQ} \geq 0,5=8$ patients $(27 \%)$. AE was present in $1(3 \%)$, SIP in 7 $(24 \%)$, LP in $6(21 \%)$, and LL in $12(41 \%)$ children. Nineteen $(65 \%)$ patients showed JADAS $>1$ and $21(76 \%)$ JSpADA $>0$. Radiologic sacroilitis was recorded in $21(72 \%)$ children. Fourteen (48\%) patients were treated with TNFi. Th1 cell percentage in ERA was $8.5 \pm 3.4 \%$ (range, 4-17.4) while healthy controls was $5.8 \pm 3.8 \%$ (range, $1.2-14.2$ ), $p=0.023$. Th17cell\% in ERA was $0.90 \pm 0.44$ (range, $0.39-2.34 \%$ ) while controls was $0.55 \pm 0.38$ (range, $0,17-1,61 \%$ ); $p=0.004$. There was no difference between T-cells\% and active/inactive disease. Eighteen (62\%) children showed peripheral pattern, while 11 (38\%) exhibited non-peripheral. Peripheral and non-peripheral groups showed Th17\%cells $0.90(0.39-1.74)$ vs $1.14(0.64-2.44)$ respectively $(p=0.018)$. Significant correlations were Th1 with AJ $(r=0.45 p=0.004)$ and Th17with LP $(r=0.83 p=0,0001), \operatorname{LL}(r=0.47 p=0.03)$.

Conclusions: Th1 and Th17 cells subsets were significantly higher in ERA compared with healthy controls. However, T-cells showed no significant difference between patients with active versus inactive disease. Interestingly, non-peripheral pattern showed higher Th17\% cells respect to patients with peripheral disease.Our results suggest that Th17 evaluation could help identify different phenotypes that benefit from Th-17 blocking strategies.

References:

[1] Biologic agents in juvenile spondyloarthropathies. Katsicas MM, Russo R.

Pediatr Rheumatol Online J.2016Mar 12;14 (1):17.

Disclosure of Interest: None declared

DOI: 10.1136/annrheumdis-2017-eular.6726

\section{THU0522 CORRELATION BETWEEN SERUM CALPROTECTIN (MRP8/14), CLINICAL AND ULTRASOUND ASSESSMENT IN PATIENTS WITH JUVENILE IDIOPATHIC ARTHRITIS}

M. Romano, V. Gerloni, M. Gattinara, O. De Lucia, P.L. Meroni. Rheumatology, G.Pini Istitute, Milan, Italy

Background: MRP8/14 (also known as calprotectin) has been widely studied as 
potential predictor of disease activity and response to treatment in inflammatory diseases. In Juvenile Idiopathic Arthritis (JIA), MRP8/14 levels are highly predictive of disease flares in systemic JIA. In a more heterogeneous group of JIA patients (pts), MRP8/14 levels have been shown to predict response to MTX. High levels of baseline MRP8/14 are associated with good response to anti-TNF treatment, whereas elevated MRP8/14 levels at time of discontinuation are associated with higher chance to flare. In clinical practice, ultrasound could be usefull to define the state of disease activity. Indeed, PD-US assessment of synovial vascularization has been shown to be more sensitive than serum markers of inflammation in the identification of active disease in JIA.

Objectives: To explore the association between calprotectin, clinical and US assessment in JIA pts.

Methods: A total of 30 consecutive pts (aged under 18 years) with oligo or poly JIA were assessed by US, clinical examination and MRP8/14 serum levels. Serum MRP8/14 was measured by ELISA in all pts and in 20 age matched healthy controls. Ultrasonographic B-mode and power Doppler assessment of 44 joints each pts were performed. Patients were evaluated using Wallace criteria.

Results: 30 consecutive non systemic JIA pts (F 18) were evaluated: 13 persistent and 8 extended oligoarticular, 6 polyarticular (1 RF positive), 2 ERA, 1 psoriatic JIA. Median age at disease onset was 10.6 yrs (mean 10.8, range 2-16). Mean disease duration was 5.4 yrs (range $0.1-15.9$ ). Mean active joints was 2 (range 0-26). 14 pts were active according to Wallace criteria and 16 pts were active according to US evaluation. Ultrasonographic B-mode and power Doppler assessment was significantly correlated with clinical examination (Mcnemar test $\mathrm{p}=0.683$, Cohen's $\mathrm{K}$ 0.602). The majority of our enrolled pts were in phase of oligoarticular involvement with minimal disease activity (median active joints $=0$ ). No statistically significant difference in serum MRP8/14 was found between all JIA pts and healthy controls $(p=0.33)$. No statistically significant difference in serum MRP8/14 was found between active (according to clinical examination) JIA pts and healthy controls $(p=0.69)$ and between inactive JIA $p t s$ and healthy control $(p=0.23)$. Concentrations of MRP8/14 in active and inactive pts according to Wallace were not significantly different $(p=0.75)$. No statistically significant difference in serum MRP8/14 was found between active and inactive pts according to US assessment (0.85). Only 6 pts (4 out of 6 with polyarticular course) showed calprotectin levels higher than normal. We found a correlation between calprotectin and CRP (Spearman r 0.4380, $p=0.01$ ) and between calprotectin and ESR (Spearman r 0.3800, $p=0.05$ ).

Conclusions: To our knowledge this is the first study to examine the correlation between MRP8/14 levels, clinical and US assessment in JIA. Serum levels of MRP8/14 (a biomarker of activation of the innate immune system) are not significatively different in oligoarticular JIA from healthy controls. Calprotectin high levels, could be related with a poliarticular disease either in clinical activity or in subclinical remission. However our study need to be extended to a larger number of pts followed prospectively.

Disclosure of Interest: None declared

DOI: 10.1136/annrheumdis-2017-eular.6951

\section{THU0523 MORTALITY AND CAUSES OF DEATH AMONG JUVENILE IDIOPATHIC ARTHRITIS PATIENTS IN FINLAND}

M. Kyllonen ${ }^{1,2}$, H. Kautiainen ${ }^{3}$, K. Puolakka ${ }^{4}$, P. Vahasalo ${ }^{1,5} .{ }^{1}$ Pedego Research Unit, Oulu University; ${ }^{2}$ Internal Medicine, Oulu University Hospital, Oulu; ${ }^{3}$ General practise and Primary health care, Helsinki University, Helsinki; ${ }^{4}$ South Karelia Central Hospital, Lappeenranta; ${ }^{5}$ Pediatrics, Oulu University Hospital, Oulu, Finland

Background: Juvenile idiopathic arhritis (JIA) is an inflammatory arthritis that begins before 16 years of age and persists more than 6 weeks. The prevalence of JIA is approximately 1 to 2 per 1,000 children. Only $40-60 \%$ of JIA patients had inactive disease at follow-up (1).

An increase rate of mortality has been observed in JIA. A follow-up study (1960-1993) in the United States reported early deaths especially in systemic JIA (2). The same was found in an follow-up study in Scotland (3). However two follow -up studies in United States and one study in Germany showed no increased mortality $(4,5,6)$.

Objectives: To explore mortality rates and causes of death in JIA patients in Finland compared with the general population.

Methods: All incidents patients with JIA during 2000-2014 were collected from the nationwide register of special reimbursements for disease-modifying antirheumatics drugs, maintained by the Social Insurance Institution of Finland. The National Population Registry identified three age, sex and residence matched controls for each case. Death certificate data up to 2015 were obtained from the Statistics of Finland.

Results: Altogether 4,180 JIA patients (62\% girls) were identified. Mean age at diagnosis was 8.3 years. The average follow-up was 6.6 years (IQR 3.1-10.5). These patients were compared with 12,511 controls.

During 28.941 follow-up years, 11 JIA patients ( 6 girls, 5 boys) and 23 controls (12 girls, 11 boys) died.

Mean age at death was 20.3 years (range 11-30) in the JIA patients and 23.1 years (range 9-29) years in the control group. $(p=0.17)$.

Cumulative mortality in the patients with JIA was $0.4 \%(95 \% \mathrm{Cl} 0.2-0.8 \%)$ compared to $0.3 \%(95 \% \mathrm{Cl} 0.2-0.5 \%)$ in the controls, hazard ratio was $1.44(95 \%$ Cl 0.70-2.95).
Accidents were the most common (54\%) cause of death in the patients with JIA, but suicide $(39 \%)$ in the controls. Alcohol, drug abuse and depression contributed more to deaths in the control (39\%) than in JIA group (1\%), $p=0.053$.

Conclusions: Juvenile idiopathic arthritis is not associated with a higher mortality rate in children and young adults.

References:

[1] Ravelli et al. Juvenile idiopathic arthritis. Lancet 2007;369: 767-78.

[2] French et al. Increased mortality in adults with a history of juvenile rheumatoid arthritis. ArthrRheum 2001:44: 523-527.

[3] Thomas et al. National study of cause-spesific mortality in rheumatoid arhtritis, juvenile chronic arthritis and other rheumatic conditions: a 20 year follow up. JRheumatology, 2003;30:958-965.

[4] Hashkes $P$ et al. Mortality outcomes in pediatric rheumatology in the US. ArthrRheum 2010;62:599-608.

[5] Krause et al. Juvenile idiopathic arthritis in Olmsted County, Minnesota 1960-2013. ArthrRheum 2016; 68: 247-254.

[6] Minden et al. Long- term outcome in patients with juvenile idiopathic arthritis ArthrRheum 2002; 46: 2392-2401.

Disclosure of Interest: None declared

DOI: 10.1136/annrheumdis-2017-eular.4042

\section{THU0524 VITAMIN D DEFICIENCY AND RISK FOR CARDIOVASCULAR DISEASE IN JUVENILE IDIOPATHIC ARTHRITIS}

M.H. Abu-Zaid ${ }^{1}$, A.M. El-Barbary ${ }^{1}$, R.A. Gaber ${ }^{2}$, H.H. Abdelnabi ${ }^{3}$. ${ }^{1}$ Rheumatology and Rehabilitation, Faculty of Medicine Tanta University Egypt; ${ }^{2}$ Biochemistry; ${ }^{3}$ Pediatric, Faculty of Medicine Tanta University, Tanta, Egypt

Background: Vitamin D is not a classic vitamin but rather a prohormone which converted primarily in the liver into its major circulating form, 25-hydroxy-vitamin $D[25(\mathrm{OH}) \mathrm{D}]$. Serum levels of vitamin $\mathrm{D}$ have been found to be low in juvenile idiopathic arthritis (JIA) as in several autoimmune diseases ${ }^{1}$. There are some evidences of an association between serum vitamin $\mathrm{D}$ and cardiovascular disease risk $^{2}$.

Objectives: To assess the association between serums levels of 25-hydroxyvitamin $\mathrm{D}$ and cardiovascular disease risk factors in juvenile idiopathic arthritis

Methods: A prospective cross-sectional study was done on 30 patients with JIA according to the criteria of the International League of Associations for Rheumatology (ILAR) and 30 healthy volunteers matched for age and gender. Patients with other causes of dyslipidemia or those whom receiving vitamin D supplements or lipid-lowering medications were excluded from this study. Systolic and diastolic blood pressure was measured to all patients and controls Also; all patients and controls underwent laboratory tests of plasma $25(\mathrm{OH}) \mathrm{D}$, Serum High-Density Lipoprotein (HDL), Low-Density Lipoprotein cholesterol (LDL). Echocardiography, carotid intima media thickness (cIMT), flow mediated dilatation of the brachial artery (FMD) also was done to all patients and controls. Vit $D$ levels were then correlated in each subgroup with the other clinical, laboratory and radiological parameters. We predefined Vit $D$ insufficiency as being $<50 \mathrm{nmol} / \mathrm{l}$ and Vit $D$ deficiency as being $<25 \mathrm{nmol} / \mathrm{l}$.

Results: The mean serum vitamin D levels of all patients were $23.8 \mathrm{nmol} / \mathrm{l}+16.59$. Only 7 patients $(23.3 \%)$ have adequate vitamin D levels $(50-75 \mathrm{nmol} / \mathrm{l})$. While Vitamin D insufficiency (serum vitamin D: $25-50 \mathrm{nmol} / \mathrm{l}$ ) and deficiency (serum vitamin $D$ level $<25 \mathrm{nmol} / \mathrm{l})$ were found in $9(30 \%)$ and 14 patients $(46.7 \%)$, respectively. JIA patients had significantly lower vitamin $D$ levels as compared to controls $(p<0.01)$. JIA patients had higher systolic and diastolic blood pressure than controls although these differences were not statistically significant $(p>0.05)$ and all levels were still in normal values. Subjects with vitamin $D$ deficiency or insufficiency had significantly lower values for HDL cholesterol and significantly elevated values for LDL cholesterol as compared to controls and patients with adequate vitamin $D$ levels, with significant positive correlation between $25(\mathrm{OH}) \mathrm{D}$ and HDL cholesterol, and significant inverse correlation between 25(OH)D and LDL levels.

Patients with lower levels of vitamin D had significantly higher CIMT and lower FMD $(p<0.01)$ With significant positive correlation between 25(OH)D and FMD, and significant inverse correlation between $25(\mathrm{OH}) \mathrm{D}$ and cIMT, while there were no significant differences in echocardiography results.

Table 1

\begin{tabular}{lcccc}
\hline Vit D levels & HDL & LDL & cIMT & FMD \\
\hline & $r=0.8493$ & $r=-0.6032$ & $r=-0.6616$ & $r=0.8527$
\end{tabular}

Conclusions: Vitamin D deficiency is a cardiovascular risk factor in juvenile idiopathic arthritis.

\section{References:}

[1] Çomak E, Doğan ÇS, Uslu-Gökçeoğlu A, Akbaş H, Özdem S, Koyun M, Akman S. Association between vitamin D deficiency and disease activity in juvenile idiopathic arthritis. Turk J Pediatr. 2014; 56(6):626-31.

[2] Suzanne E. Judd and Vin Tangpricha. Vitamin D Deficiency and Risk for Cardiovascular Disease. Am J Med Sci. 2009; 338(1): 40-44.

Acknowledgements: Thanks to Dr. Samar Abd Alhamed for her efforts in completing statistics in this study.

Disclosure of Interest: None declared

DOI: 10.1136/annrheumdis-2017-eular.1214 